\title{
Acute massive pulmonary embolism without deep venous thrombosis
}

Özge Korkmaz, ${ }^{1}$ M. Birhan Yılmaz, ${ }^{2}$ Sabahattin Göksel, ${ }^{1}$ Müslim Gül, ${ }^{3}$ Öcal Berkan ${ }^{1}$

Received: July 08, 2014 Accepted: December 29, 2014 Published online: February 10, 2015

Acute pulmonary embolism is rarely encountered following coronary artery bypass graft (CABG) surgery. Herein, we present a 75 -yearold woman who developed an acute massive pulmonary embolism without deep venous thrombosis 20 days after four-vessel CABG surgery. She was diagnosed using a thoracic computed tomography scan, yielding subtotal occlusion of the right and left pulmonary arteries. She was administered successful thrombolytic therapy and discharged uneventfully without problems during the follow-up period.

Keywords: Computed tomography; coronary artery bypass grafting; deep venous thrombosis; pulmonary embolism.

Acute pulmonary embolism (PE) following cardiac bypass surgery is an uncommon complication, yet leads to potentially fatal complications. It is known to increase morbidity and mortality. The estimated incidence of acute $\mathrm{PE}$ ranges between $0.5 \%$ and $4 \% .{ }^{[1]}$ The diagnosis is often challenging as symptoms such as shortness of breath, chest pain, hypoxia, and leg swelling are often attributed to pre-procedural changes. Therefore, the diagnosis of $\mathrm{PE}$ or deep vein thrombosis (DVT) may be challenging. Furthermore, on occasion, DVT may not accompany acute PE after coronary artery bypass graft (CABG). In the light of the current literature data, we present a case who developed acute $\mathrm{PE}$ after $\mathrm{CABG}$ in the absence of detectable DVT along with successful recovery after thrombolytic therapy.

\section{CASE REPORT}

A 75-year-old woman (body mass index <20) who did not have accompanying diabetes mellitus or hyperlipidemia, underwent coronary angiography which showed serious lesions of the left anterior descending artery, right coronary artery, and circumflex coronary artery after presenting with unstable angina pectoris. According her medical story, she was on a beta blocker for hypertension. She underwent a four-vessel CABG with the use left internal mammarian artery for grafting the left anterior descending artery, and saphenous vein graft which was harvested using a classic method for grafting first branch of obtuse marginal, first branch of diagonal and right coronary artery. The surgical procedure was not complicated with a cross-clamp time of 79 minutes and with a cardiopulmonary bypass time of 116 minutes. During the first operative night, all vital parameters were steady and there were no complications. On the first postoperative day, she was transferred to the ward. She was administered a daily dose of 8000 IU low molecular weight heparin in addition to $100 \mathrm{mg}$ of acetyl salicylic acid and $75 \mathrm{mg}$ of clopidogrel. She was discharged on the $10^{\text {th }}$ postoperative day. Prior to discharge, she was prescribed acetyl salicylic acid, clopidogrel, metoprolol, atorvastatin and ramipril. Twenty days postoperatively, the patient was admitted to the emergency department with shortness of breath and chest pain, which started four hours earlier. On admission, vital parameters were as follows: respiratory rate $35 /$ minute, heart rate $135 /$ minute, and blood pressure $85 / 45 \mathrm{mmHg}$ with moderate oxygen desaturation (85\%), and cold sweats. Echocardiography showed sinus tachycardia along with negative $\mathrm{T}$ waves on the anterior leads. Transthoracic echocardiography was performed immediately and revealed mild right ventricular (RV) dilatation with hypocontractility in the interventricular septum. A differential diagnosis of

\footnotetext{
${ }^{1}$ Department of Cardiovascular Surgery, Medical Faculty of Cumhuriyet University, Sivas, Turkey

${ }^{2}$ Department of Cardiology, Medical Faculty of Cumhuriyet University, Sivas, Turkey ${ }^{3}$ Department of Cardiovascular Surgery, Sivas Numune Hospital, Sivas, Turkey
}

Corresponding author: Özge Korkmaz, M.D. Cumhuriyet Üniversitesi Tıp Fakültesi, Kalp ve Damar Cerrahisi Anabilim Dalı, 58140 Sivas, Turkey.

Tel: +90 532 - 5922091 e-mail: ozgekorkmaz73@hotmail.com 


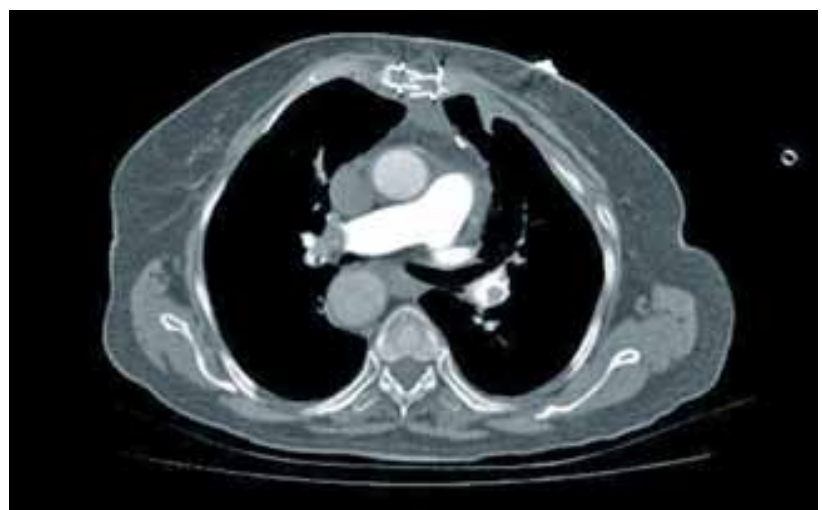

Figure 1. Contrast-enhanced transaxial spiral computed tomographic scan at the level of contain the both left and right main pulmonary. Scan acquired during late arterial phase of contrast enhancement.

acute coronary syndrome (ACS) versus acute PE was considered. Therefore, cardiac enzymes and D-dimer were studied. Troponin level was $0.19 \mathrm{ng} / \mathrm{mL}$ and D-dimer level was $17.48 \mathrm{ng} / \mathrm{mL}$. In the light of these findings, an emergent thoracic computed tomography (CT) scan was sought and it confirmed subtotal occlusion of the right and left pulmonary arteries (Figure 1). In the meantime, she had similar ECG and echocardiographic abnormalities in the previous records, postoperatively; hence, the diagnosis of ACS was eliminated. In addition, her deep venous system was investigated using Doppler ultrasonography which produced negative results. Since her hemodynamic condition was not improving, she was referred for thrombolytic therapy of $0.6 \mathrm{mg} / \mathrm{kg}$ tissue plasminogen activator (tPA). No complications were detected during this protocol. During in-hospital follow-up, troponin levels slightly increased, then decreased sharply within 24 hours, while the level of D-dimer declined slowly within four days. She was hospitalized for 10 days and no complication was seen. She was discharged with warfarin, acetyl salicylic acid, metoprolol, atorvastatin, and ramipril. She was scheduled for visit two weeks later after discharge.

\section{DISCUSSION}

Previous retrospective studies reported that acute PE was an uncommon complication with a maximum incidence of $0.6 \% .^{[2]}$ On the other hand, most of recent studies introduced an incidence of acute $\mathrm{PE}$ that is higher than the previous reports with a range between $2.7 \%$ and $3.9 \% .^{[2]}$ Acute $\mathrm{PE}$ is a life-threatening condition. Unfortunately, the presentation after $\mathrm{CABG}$ may be deceptive. Most patients, as in our case, suffer from ambiguous symptoms which are not helpful in the tentative diagnosis of acute PE after CABG. Moreover, these findings do not help the differential diagnosis of acute myocardial infarction or congestive heart failure. Even measuring D-dimer level and the cardiac enzyme profile may not be helpful for a definitive diagnosis. In addition, echocardiography is not useful in the differential diagnosis due to preexisting wall motion abnormalities, either due to CABG itself of due to previous infarctions, which may cause some confusion, though, severe right ventricular dilatation may help in case of hemodynamically significant $\mathrm{PE} .{ }^{[3]}$ In this present case, the diagnosis was confirmed with a CT scan due to the fact that multi-slice CT, which was able to accurately make the differential diagnosis, was not available in the emergency department setting.

Recent guidelines recommend thrombolytic therapy in patients having acute massive acute $\mathrm{PE}$ with a high risk for early death. ${ }^{[4]} \mathrm{A}$ number of controlled clinical trials confirm streptokinase, urokinase or alteplase therapy for patients suffering from significant acute PE. Currently, there is a consensus that patients with massive acute $\mathrm{PE}$ presenting with serious right ventricular failure with or without clinical instability and cardiogenic shock should be treated with thrombolytic agents expeditiously. ${ }^{[5]}$ Hence, in our case, as soon as the definitive diagnosis was made, thrombolytic therapy of $0.6 \mathrm{mg} / \mathrm{kg}$ tPA was dispensed within one hour in the intensive care unit. After therapy, she was administered warfarin and low-molecular weight heparin. Symptoms and hemodynamic status recovered remarkably in 48 hours.

In conclusion, although acute $\mathrm{PE}$ is an uncommon complication after cardiac surgery, the rate of incidence is higher than expected. Early diagnosis and rapidly tailored medical therapy is crucial. For the differential diagnosis of acute PE accurately, the gold standard remains to be thoracic CT scanning, particularly if a triple scan is available with multi-slice CT.

\section{Declaration of conflicting interests}

The authors declared no conflicts of interest with respect to the authorship and/or publication of this article. 


\section{Funding}

The authors received no financial support for the research and/or authorship of this article.

\section{REFERENCES}

1. Close V, Purohit M, Tanos M, Hunter S. Should patients post-cardiac surgery be given low molecular weight heparin for deep vein thrombosis prophylaxis? Interact Cardiovasc Thorac Surg 2006;5:624-9.

2. Protopapas $\mathrm{AD}$, Baig $\mathrm{K}$, Mukherjee $\mathrm{D}$, Athanasiou $\mathrm{T}$.
Pulmonary embolism following coronary artery bypass grafting. J Card Surg 2011;26:181-8.

3. Wood KE. Major pulmonary embolism: review of a pathophysiologic approach to the golden hour of hemodynamically significant pulmonary embolism. Chest 2002;121:877-905.

4. Lankeit M, Konstantinides S. Thrombolytic therapy for submassive pulmonary embolism. Best Pract Res Clin Haematol 2012;25:379-89.

5. Konstantinides S. Should thrombolytic therapy be used in patients with pulmonary embolism? Am J Cardiovasc Drugs 2004;4:69-74. 